\title{
Prospects for elUCidating the PHYLOgeny Of the APICOMPLeXA
}

\author{
MORRISON D.A.*
}

\section{Summary:}

The Apicomplexa is traditionally considered to contain four clearly defined groups, but the phylogenetic relationships among these groups, and their subsequent taxonomic arrangement, are currently unclear. Here, I consider five of the important criteria that need to be met before radical taxonomic changes are made, in relation to phylogenetic analyses. Unfortunately, at least four of these criteria indicate that the prospects for elucidating the phylogeny and taxonomy of the Apicomplexa are not good in the short term. A lot more pertinent data need to be collected, both in terms of taxa sampled and genes sequenced, before any concordance among phylogenies is likely to emerge; and someone (e.g. a consortium) needs to co-ordinate the active collection and analysis of these data if any worthwhile progress is to be made.

KEY WORDS : Coccidia, Piroplasma, Haemosporidia, Gregarina.

T The sporozoans (phylum Apicomplexa) form a diverse group of unicellular parasitic protists, whose motile invasive stages are characterized by the presence of an evolutionarily unique apical complex. The group contains approximately 6,000 named species, with perhaps only $0.1 \%$ of the species having been named to date (Adl et al., 2007), many of which are pathogens of humans and domesticated animals. The phylum is now recognized as being closely related to the dinoflagellates and ciliates (forming the taxonomic group Alveolata), and less closely to the stramenopiles (forming the group Chromalveolata) (Simpson \& Roger, 2004; Adl et al., 2005; Keeling et al., 2005).

Apicomplexa is traditionally considered to contain four clearly defined groups (Adl et al., 2005): the coccidians, the gregarines, the haemosporidians, and the piroplasms. These groups are based largely on phenotypic characteristics, such as host, tissue and vector specificities, and were originally designed to be utilitarian rather than phylogenetic (Ellis et al., 1998; Tenter et al., 2002). Thus, the evolutionary relationships among the four groups, and their subsequent taxonomic arrangement, are currently unclear.

\footnotetext{
* Department of Parasitology (SWEPAR), National Veterinary Institute and Swedish University of Agricultural Sciences, 75189 Uppsala, Sweden.

Tel.: +4618 674161 - Fax: +4618309162.

E-mail: David.Morrison@bvf.slu.se
}

This is unfortunate, because the scientific study of diversity and evolution requires a stable taxonomy based on a robust phylogeny. The groups named in the taxonomy should represent real evolutionary groups (called clades), so that the groups can be used to identify the pertinent experimental comparisons that are needed for the quantitative study of both diversity patterns and evolutionary processes.

In recent years, phylogenetic evidence provided by molecular data has lead many biologists to question traditional taxonomic schemes, and to propose changes that are often quite dramatic; and this has been as true of the Apicomplexa as it has elsewhere. Widely adopted higher-level taxonomic schemes are neither forced upon scientists as a group nor are they ratified by formal consensus. Instead, such schemes are usually sanctioned because a majority of those people who have considered the evidence in their favor have found it convincing (Entwisle \& Weston, 2005). It is therefore the acquisition of convincing evidence that is the key to a stable taxonomic scheme; and if taxonomy is to reflect phylogeny then it is phylogenetic evidence that is the crucial evidence. It is therefore instructive to consider the important criteria that have been shown need to be met before radical taxonomic changes are made, in relation to phylogenetic analyses.

There seem to be a number of significant features relating phylogenetic analyses to stable taxonomic changes, including: 1) taxon sampling needs to be adequate, in order to provide a convincing case for particular taxonomic boundaries; 2) there needs to be concordance between the phylogenies derived from several molecular sequences, preferably at least one of them nuclear and one organellar; 3) phylogenetic analyses need to be thorough, so that the evolutionary relationships are not artifacts of the analysis procedure; 4 ) it needs to be possible to re-interpret the homologies previously proposed for any contradictory phenotypic characters so that they agree with the new phylogeny; 5) there needs to be a widespread base of people collating the molecular data for the chosen gene sequences, and a consortium (or perhaps a small informal group of people) needs to take responsibility for the compilation and analysis of the necessary data. 
These are not prescriptions for what must happen, but merely personal observations about what has happened in practice in better-studied taxonomic groups, notably fungi and flowering plants. Unfortunately, at least four of these criteria indicate that the prospects for elucidating the phylogeny and taxonomy of the Apicomplexa are not good in the short term.

\section{TAXON SAMPLING}

axon sampling needs to be adequate in order to provide a convincing case for particular taxonomic boundaries. Showing that a problem exists is easy with a small sample size, but revealing the solution takes much more effort. Unfortunately, taxon sampling for the Apicomplexa is woeful for most molecular sequences, as phylogenetic trees based on molecular data have been produced for only a small subset of the known species.

There are more than 300 recognized genera, in at least 60 families (Perkins et al., 2000), from which to choose exemplar taxa for phylogenetic analysis, but the ones chosen to date have usually been based on their veterinary or medical importance. That is, economics has been the criterion of choice rather than phylogenetic relevance, which leads to a small and biased sample. The GenBank database currently contains nucleotide sequence data for only a few members of the Apicomplexa (Table I). There is a major bias towards just five genera: Plasmodium (131,164 sequences), Cryptosporidium (13,785), Theileria (8,735), Toxoplasma $(7,464)$ and Babesia (4,873), which account for $98 \%$ of the sequences. There are relatively few sequences for some of the largest genera, such as: Eimeria, Gregarina, Haemogregarina, Haemoproteus, Isospora and Sarcocystis. Furthermore, the representation of the gregarines as a whole is very poor (Leander et al., 2003). A lot more pertinent data need to be collected in terms of widespread taxon sampling, before any reliable phylogenies are likely to emerge.
Another obvious problem is the lack of sequence data for potential basal groups (i.e. close to or within the Apicomplexa), such as: Blastocystea (0 sequence), Colpodella (6) and Metchnikovellea (0). Perhaps the only worthwhile contribution, to date, of molecular data to the base of the Apicomplexan tree is the clear exclusion of Perkinsus from the phylum (Siddall et al., 2001; Kuvardina et al., 2002; Saldarriaga et al., 2003). Basal taxa are vital for the rooting of the phylogenetic tree, which then determines the relationships among the other taxa, and thus the definition of the clades. It is the clades that form the groups to be recognized in the taxonomy. Outgroups should consist of basal members of the sister group to the clade being studied (i.e. the taxa that are most similar to the ancestor of the ingroup); choice of more distant outgroup taxa merely results in random rooting of the ingroup tree.

Nevertheless, interesting patterns have already emerged from the available data. For example, the traditional definition of some of the larger genera makes them undoubtedly paraphyletic (e.g. Babesia, Eimeria, Plasmodium) or polyphyletic (e.g. Isospora) (Ellis et al., 1998; Perkins \& Schall, 2002; Morrison et al., 2004). Clearly, there will be some major re-definition of taxonomic groups throughout the Apicomplexa. Furthermore, even some smaller genera are rendered paraphyletic by the recognition of economically important pathogens as separate genera; for example, the relationship between Hammondia and both Neospora caninum and Toxoplasma gondii (Monteiro et al., 2007). This can lead to serious conflicts between the scientific requirements of classification and the more pragmatic requirements of end-users (Šlapeta et al., 2002; Pérez-Tris et al., 2005).

\section{MULTIPLE MOLECULAR DATA SETS}

f taxonomy is to reflect phylogeny, then we need to reconstruct the phylogeny of the species involved. A cladogram from a single molecular sequence

\begin{tabular}{|c|c|c|c|c|}
\hline \multirow[b]{2}{*}{ Group } & \multicolumn{4}{|c|}{ Number of } \\
\hline & Speciesa & $\begin{array}{l}\text { Nucleotide } \\
\text { sequences }^{b}\end{array}$ & $\begin{array}{c}\text { Nuclear } \\
\text { genomes }\end{array}$ & $\begin{array}{c}\text { Other } \\
\text { genomes }^{d}\end{array}$ \\
\hline Haemosporines & 600 & 131,752 & $6(4)$ & 8 \\
\hline Piroplasms & 150 & 13,643 & $1(2)$ & $0(1)$ \\
\hline Archigregarines & 15 & 0 & 0 & 0 \\
\hline Eugregarines & 1,700 & 44 & 0 & 0 \\
\hline Neogregarines & 60 & 4 & 0 & 0 \\
\hline Agamococcidians & 5 & 1 & 0 & 0 \\
\hline Protococcidians & 115 & 0 & 0 & 0 \\
\hline Adeleorines (inc. Haemogregarines) & 500 & 102 & 0 & 0 \\
\hline Eimeriorines (inc. Cryptosporidium) & 1,800 & 23,968 & $3(3)$ & $0(2)$ \\
\hline
\end{tabular}

a Based on Perkins et al. (2000); b NCBI GenBank, as at 24 September 2007; c Complete (in progress); d Mitochondrion (apicoplast).

Table I. - Available molecular data for the major groups within the Apicomplexa. 
represents only the phylogeny of that one gene, which is not necessarily the phylogeny of the species. Thus, there needs to be concordance between the phylogenies derived from several molecular sequences, preferably at least one of them nuclear and one organellar. Only concordance will be accepted as evidence for the species phylogeny rather than the gene phylogeny. For the Apicomplexa, molecular phylogenies have usually been based on the nucleotide sequence of the gene for the small subunit ribosomal RNA (18S rRNA) (e.g. Perkins \& Keller, 2001; Morrison et al., 2004; Kopečná et al., 2006; Leander et al., 2006), which is the most widely studied gene sequence for most phyla. Unfortunately, there can be serious problems with paralogy of this gene within the Apicomplexan, as many taxa have been reported to have a small number of divergent copies (rather than a large number of copies homogenized by concerted evolution), which can confound phylogenetic analyses (Morrison, 2005).

Nevertheless, sequences of multiple protein-coding genes from the same taxa are slowly becoming available, notably for the nuclear actin, $\alpha$-tubulin, $\beta$-tubulin and HSP70 genes, and the mitochondrial cox1 and cytb genes. Unfortunately, there is a tendency to use only fragments of these genes (e.g. Schrenzel et al., 2005), which may provide very little data and also makes comparisons between studies difficult. Furthermore, many of the other gene sequences have been surface proteins, sequenced as part of drug/vaccine projects, and these often have restricted taxonomic distributions (Templeton, 2007). Nevertheless, multi-gene phylogenies are starting to appear for a number of genera, including Cryptosporidium (Xiao et al., 2002; Morrison, 2006a) and Plasmodium (Rathore et al., 2001; Perkins et al., 2007). Unsurprisingly, very few nuclear genomes have yet been sequenced within the Apicomplexa (Table I), in contrast to the much better-studied fungi (e.g. Fitzpatrick et al., 2006). So, genomes have not yet made any worthwhile contribution to Apicomplexan phylogenetic analyses, even though they have contributed to comparative genomics (which assumes that the phylogeny is known). Similarly, although there is now a large amount of EST data available (Li et al., 2004), the taxon sampling is still too limited for meaningful phylogenetic analysis.

Perhaps more surprisingly, there are also very few organellar genomes available (Table I), either mitochondrial or from the Apicomplexa-specific apicoplast (Waller \& McFadden, 2005). Organellar genomes provide a different perspective on the evolutionary history of a taxon (e.g. usually with maternal inheritance), and so they can be seen as a useful adjunct to nuclear information when trying to produce robust phylogenies (Page \& Holmes, 1998). Unfortunately, in many of the popularly studied Apicomplexan taxa the organellar genomes may be reduced (e.g. Plasmodium) or absent (e.g. Crypto- sporidium), with the associated genes having become incorporated into the nuclear genome (Huang et al., 2004). Moreover, the plastid rRNA genes are duplicated (part of an inverted repeat), while the mitochondrial rRNA genes may be fragmented, both of which lead to problems determining homology (Perkins et al., 2007).

\section{PHYLOGENETIC ANALYSES}

$\mathrm{P}$ hylogenetic analysis of molecular sequences usually consists of two distinct procedures: sequence alignment and tree building. It is possible to do basic analyses for both procedures simply by choosing some popular computer program (e.g. Clustal and PAUP*, respectively) and then using the default parameter values of that program. However, this is a very naïve approach, because it does not consider the possible unsuitability of the analyses for the data at hand, which may lead to results that are artifacts. There are many known potential artifacts for both sequence alignment (reviewed by Morrison, 2006b) and tree building (reviewed by Morrison, 2006a) that thus need to be considered seriously in all analyses.

For sequence alignment, one of the biggest problems is that sequence similarity decreases rapidly as the taxa become more distant, so that processes causing sequence length variation become more probable (e.g. duplication, translocation, deletion, insertion and inversion). This exacerbates the problems of poor taxon sampling, particularly for rRNA genes, which are very lengthvariable even at the best of times. It also exacerbates the problems caused by distant outgroups, which can be very difficult to align with the ingroup.

For tree building, compositional biases are a recurring problem. Within the Apicomplexa, this is particularly troublesome for Plasmodium, which has a severely ATrich genome compared to most other taxa (Morrison et al., 2004; Morrison, 2006a), and for Aggregata, which has a very low C content (Kopečná et al., 2006). Also, juxtaposed long and short branches are now known to be problematic. Long branches, associated with increased rates of evolutionary change, have been observed in Aggregata, Cryptosporidium, Plasmodium and several eugregarines. However, they also occur when there is a distant outgroup, as the branch connecting the ingroup and outgroup will be much longer than the within-ingroup branches (e.g. Schrenzel et al., 2005; Monteiro et al., 2007). It is worth noting that the common practice of performing multiple analyses of the same data set (e.g. a parsimony analysis and a maximum-likelihood analysis) will not successfully deal with either of these two problems, as all of the analyses will be affected in the same way. Other potential problems are reviewed by Roger \& Hug (2006). This does not mean that the results of basic and more sophisticated analyses must necessarily disagree. For 
example, the analyses of the same dataset by Xiao et al. (2002) and Morrison (2006a) produced generally congruent results, although the latter identified many ways in which the data violate the assumptions of the various analyses.

\section{RE-INTERPRETATION OF HOMOLOGIES}

A fter a phylogenetic analysis of molecular data, it needs to be possible to re-interpret the homologies previously proposed for any contradictory phenotypic characters, so that they are consistent with the new phylogeny (Taylor, 1999). Such re-interpretation might involve the realization that some of the characters are functionally or developmentally correlated, rather than being independently informative phylogenetically. Alternatively, the phenotypic data might have been over-simplified, by combining several independent characters into one complex character; or the data set may have been incomplete or inaccurate. Within the Apicomplexa, molecular data indicate that Hammondia hammondi is closely related to Toxoplasma gondii while Hammondia heydorni is related to Neospora caninum (Monteiro et al., 2007). Therefore, the characters upon which these three genera were originally based need to be re-evaluated, including the location of the tissue cysts (brain versus muscles) and the type of heteroxeny (facultative versus obligate); and both of these characters have been successfully re-evaluated. Similar re-interpretations have also occurred for the phenotypic data of other genera such as Colpodella \& Perkinsus (Siddall et al., 2001; Kuvardina et al., 2002) and Cryptosporidium \& Isospora (Tenter et al., 2002). For example, the ineffectiveness on Cryptosporidium of anti-coccidial agents is easily explained if it is not part of the Coccidia, as has been repeatedly shown (Barta \& Thompson, 2006).

Failure of any such re-interpretation, or disagreement about the re-interpretation, greatly weakens the evidence provided by gene sequences (Taylor, 1999). It is therefore fortunate that re-interpretation has so far proved to be quite successful within the Apicomplexa.

\section{DIRECTED DATA COLLECTION}

here needs to be a widespread base of people actively collating the molecular data for the cho-

1 sen gene sequences. Without this base both the taxon and character sampling will be inadequate, in the sense that data will not be available for the critical exemplar taxa. This leads to uncertainties about taxon boundaries, and concordance of multiple gene sequences cannot be demonstrated.
Taxon sampling in parasitology is usually opportunistic (Barta, 2001), which clearly cannot create either a statistically representative sample or a purposive sample. At the moment, the collection of pertinent data for the Apicomplexa can best be described as haphazard, which is unlikely to be of much practical value phylogenetically. In most cases, with the notable exception of the 18S rRNA gene, the data have not been collected with a phylogenetic analysis in mind. This means that the critical taxa have not necessarily been the focus of data collection, which they must be if phylogenetic and taxonomic problems are to be resolved.

Fortuitously available data are unlikely to be of value for phylogenetic analysis, except by random chance. It is irrelevant that cultures of the critical taxa are often hard to come by, because the taxonomic problems will not be solved until these taxa are sequenced. A lot more data need to be collected in terms of genes sequenced for critical taxa, before any concordance among phylogenies is likely to emerge.

From a purely practical point of view, some relatively small informal group of people needs to take responsibility for the compilation and analysis of the necessary data, rather than continuing with often contradictory phylogenies (and taxonomic proposals) based on ad hoc analyses of fortuitously available data. A formal group can lead to the perception of autocracy, while a large group is unnecessary and is often counter-productive. Informal groups have worked successfully within the flowering plants, notably the Angiosperm Phylogeny Group (2003) and the Grass Phylogeny Working Group (2001), both of which have produced robust phylogenies and re-classifications based on multiple gene sequences from a large set of exemplar taxa. There are similar initiatives ongoing in other taxonomic groups (e.g. fungi: Spatafora, 2005). However, to date, only one such call has explicitly been made within the Apicomplexa, for the Coccidia (Tenter et al., 2002).

\section{CONCLUSION}

onvincing evidence for a well-resolved phylogeny of the Apicomplexa, and thus a stable classification, has yet to appear. A lot more pertinent data need to be collected, both in terms of taxon sampling and genes sequenced, before any concordance among phylogenies is likely to emerge. Someone needs to co-ordinate the active collection and analysis of these data, if any worthwhile progress is to be made.

\section{REFERENCES}

Adl S.M., Leander B.S., Simpson A.G.B. et al. (17 co-authors). Diversity, nomenclature, and taxonomy of protists. Systematic Biology, 2007, 56, 684-689. 
Adl S.M., Simpson A.G.B., Farmer M.A. et al. (25 co-authors). The new higher level classification of eukaryotes with emphasis on the taxonomy of protists. Journal of Eukaryotic Microbiology, 2005, 52, 399-451.

Angiosperm Phylogeny Group. An update of the Angiosperm Phylogeny Group classification for the orders and families of flowering plants: APG II. Botanical Journal of the Linnean Society, 2003, 141, 399-436.

BARTA J.R. Molecular approaches for inferring evolutionary relationships among protistan parasites. Veterinary Parasitology, 2001, 101, 175-186.

BARTA J.R. \& ThOMPSON R.C.A. What is Cryptosporidium? Reappraising its biology and phylogenetic affinities. Trends in Parasitology, 2006, 22, 463-468.

Ellis J.T., Morrison D.A. \& Jefrries A.C. The phylum Apicomplexa: an update on the molecular phylogeny, in: Evolutionary Relationships Among Protozoa. Coombs G.H., Vickerman K., Sleigh M.A. \& Warren A. (eds), Kluwer, Dordrecht, 1998, 255-274.

ENTwisle T.J. \& Weston P.H. Majority rules, when systematists disagree. Australian Systematic Botany, 2005, 18, 16.

Fitzpatrick D.A, Logue M.E., Stajich J.E. \& Butler G. A fungal phylogeny based on 42 complete genomes derived from supertree and combined gene analysis. BMC Evolutionary Biology, 2006, 6, 99.

Grass Phylogeny Working Group. Phylogeny and subfamilial classification of the grasses (Poaceae). Annals of the Missouri Botanical Garden, 2001, 88, 373-457.

Huang J., Mullapudi N., Sicheritz-Ponten T. \& Kissinger J.C. A first glimpse into the pattern and scale of gene transfer in the Apicomplexa. International Journal for Parasitology, 2004, 34, 265-274.

Keeling P.J., Burger G., Durnford D.G., Lang B.F., Lee R.W., Pearlman R.E., Roger A.J. \& Gray M.W. The tree of eukaryotes. Trends in Ecology and Evolution, 2005, 20, 670-676.

Kopečná J., Jirki̊ M., OBorník M., TOKarevc Y.S., Lukě̌ J. \& MODRÝ D. Phylogenetic analysis of coccidian parasites from invertebrates: search for missing links. Protist, 2006, 157, 173-183.

Kuvardina O.N., Leander B.S., Aleshin V.V., Myl'nikov A.P., Keeling P.J. \& Simdyanov T.G. The phylogeny of colpodellids (Alveolata) using small subunit rRNA gene sequences suggests they are the free-living sister group to apicomplexans. Journal of Eukaryotic Microbiology, 2002, 49, 498-504.

Leander B.S., Clopton R.E. \& Keeling P.J. Phylogeny of gregarines (Apicomplexa) as inferred from small-subunit rDNA and $\beta$-tubulin. International Journal of Systematic and Evolutionary Microbiology, 2003, 53, 345-354.

Leander B.S., Lloyd S.A.J., Marshall W. \& Landers S.C. Phylogeny of marine gregarines (Apicomplexa) - Pterospora, Lithocystis and Lankesteria - and the origin(s) of coelomic parasitism. Protist, 2006, 157, 45-60.

Li L., Crabtree J., Fischer S., Pinney D., Stoeckert C.J., Sibley L.D. \& Roos D.S. ApiEST-DB, analyzing clustered EST data of the apicomplexan parasites. Nucleic Acids Research, 2004, 32, D326-D328.
Monteiro R.M., Richtzenhain L.J., Pena H.F.J., Souza S.L.P., Funada M.R., Gennari S.M., Dubey J.P., Sreekumar C., Keid L.B. \& SOARes R.M. Molecular phylogenetic analysis in Hammondia-like organisms based on partial Hsp70 coding sequences. Parasitology, 2007, 134, 1195-1203.

Morrison D.A. Networks in phylogenetic analysis: new tools for population biology. International Journal for Parasitology, 2005, 35, 567-582.

Morrison D.A. Phylogenetic analyses of parasites in the new millennium. Advances in Parasitology, 2006a, 63, 1-124.

Morrison D.A. Multiple sequence alignment for phylogenetic purposes. Australian Systematic Botany, 2006b, 19, 479-539.

Morrison D.A., Bornstein S., Thebo P., Wernery U., Kinne J. \& Mattsson J.G. The current status of the small subunit rRNA phylogeny of the Coccidia (Sporozoa). International Journal for Parasitology, 2004, 34, 501-514.

Page R.D.M. \& Holmes E.C. Molecular Evolution: a Phylogenetic Approach. Blackwell Science, Oxford, 1998.

Pérez-Tris J., Hasselquist D., Hellgren O., Krizanauskiene A., WaLdenström J. \& Bensch S. What are malaria parasites? Trends in Parasitology, 2005, 21, 209-211.

Perkins F.O., Barta J.R., Clopton R.E., Peirce M.A. \& Upton S.J. Phylum Apicomplexa Levine, 1970, in: An Illustrated Guide to the Protozoa, $2^{\text {nd }}$ edn, Vol. 1. Lee J.J., Leedale G.F. \& Bradbury P. (eds), Society of Protozoologists, Lawrence KS, 2000, 190-369.

PERKINS S.L. \& KelLER A.K. Phylogeny of nuclear small subunit rRNA genes of hemogregarines amplified with specific primers. Journal of Parasitology, 2001, 87, 870-876.

PERKINS S.L., SARKar I.N. \& CARTER R. The phylogeny of rodent malaria parasites, simultaneous analysis across three genomes. Infection, Genetics and Evolution, 2007, 7, 74-83.

Perkins S.L. \& Schall J.J. A molecular phylogeny of malarial parasites recovered from cytochrome $\mathrm{b}$ gene sequences. Journal of Parasitology, 2002, 89, 972-978.

Rathore D., Wahl A.M., Sullivan M. \& McCutchan T.F. A phylogenetic comparison of gene trees constructed from plastid, mitochondrial and genomic DNA of Plasmodium species. Molecular and Biochemical Parasitology, 2001, 114, 89-94.

Roger A.J. \& Hug L.A. The origin and diversification of eukaryotes: problems with molecular phylogenetics and molecular clock estimation. Philosophical Transactions of the Royal Society B, 2006, 361, 1039-1054.

Saldarriaga J.F., McEwan M.L., Fast N.M., TaYlor F.J.R. \& KeELING P.J. Multiple protein phylogenies show that Oxyrrbis marina and Perkinsus marinus are early branches of the dinoflagellate lineage. International Journal of Systematic and Evolutionary Microbiology, 2003, 53, 355-365.

Schrenzel M.D., Maalouf G.A., Gaffney P.M., Tokarz D., Keener L.L., McClure D., Griffey S., McAloose D. \& Rideout B.A. Molecular characterization of isosporoid Coccidia (Isospora and Atoxoplasma spp.) in passerine birds. Journal of Parasitology, 2005, 91, 635-647.

Siddall M.E., ReEce K.S., Nerad T.A. \& Burreson E.M. Molecular determination of the phylogenetic position of a species in the genus Colpodella (Alveolata). American Museum Novitates, 2001, 3314, 1-10. 
Simpson A.G.B. \& Roger A.J. The real "kingdoms" of eukaryotes. Current Biology, 2004, 14, R693-R696.

ŠLapeta J.R., Modrý D., Kyselová I., Hořejš R., LuKEš J. \& Koudela B. Dog shedding oocysts of Neospora caninum: PCR diagnosis and molecular phylogenetic approach. Veterinary Parasitology, 2002, 109, 157-167.

Spatafora J.W. Assembling the fungal tree of life (AFTOL). Mycological Research, 2005, 109, 755-756.

TAYLOR F.J.R. Ultrastructure as a control for protistan molecular phylogeny. American Naturalist, 1999, 154, S125S136.

Templeton T.J. Whole-genome natural histories of apicomplexan surface proteins. Trends in Parasitology, 2007, 23, 205-212.

Tenter A.M., Barta J.R., Beveridge I., Duszynski D.W., MehlHORn H., Morrison D.A., Thompson R.C.A. \& Conrad P.A. The conceptual basis for a new classification of the coccidia. International Journal for Parasitology, 2002, 32, 595616.

WALLER R.F. \& McFadden G.I. The apicoplast, a review of the derived plastid of apicomplexan parasites. Current Issues in Molecular Biology, 2005, 7, 57-80.

XiaO L., Sulaiman I.M., Ryan U.M., Zhou L., Atwill E.R., Tischler M.L., Zhang X., Fayer R. \& Lal A.A. Host adaptation and host-parasite co-evolution in Cryptosporidium: implications for taxonomy and public health. International Journal for Parasitology, 2002, 32, 1773-1785. 